\section{PWE-094 HEPATITIS-C INFORMATION CARDS DISTRIBUTED THROUGH COMMUNITY PHARMACIES ARE INEFFECTIVE IN INCREASING HCV TESTING AMONGST PWID}

Anja St. Clair Jones*. BSUH NHS Trust, Brighton, UK

\subsection{6/gutjnl-2018-BSGAbstracts.236}

Introduction Despite being a high risk group for hepatitis $\mathrm{C}$ virus (HCV) infection, People Who Inject Drugs (PWID) do not engage with health services. We present a low-cost intervention of issuing $\mathrm{HCV}$ information cards through community pharmacies without additional resource support to raise awareness of HCV testing, the new direct acting antivirals (DAA) and to increase self-referral of PWID to Substance Misuse Services (SMS).

Methods Brighton has a well developed and integrated community HCV clinic based at SMS.

1. Pharmacies in Brighton and Hove providing opioid substitution therapy (OST) and needle exchange were recruited

2. Cards explaining need for HCV testing, availability of DAA and contact information for community HCV nurse were provided during issue of all OST and needle exchange. A leaflet for pharmacy staff to support training needs was supplied to each pharmacy

3. Pharmacies were contacted via telephone after 1 month to obtain feedback

4. Record of self-referral was collected during 1 month

\section{Results}

1. 21 Pharmacies were recruited and participated in the project

2. 1415 cards were given to the pharmacies of which 950 were issued to clients

3. 17 pharmacies provided feed back

- All pharmacists supported this initiative though due to lack of resources were unable to allocate additional time to reinforce the message to clients

- A considerable number of longterm OST clients had already been tested as they were in contact with SMS. Some raised concerns about testing as they linked testing to monitoring of their OST

- Transient and newly started OST clients were more difficult to engage as the relationship with the pharmacy had not sufficiently evolved

- The needle exchange clients were difficult to engage and often refused the card

- One pharmacy was able to provide the intervention as part of their counselling sessions to some of the clients and found increased engagement in this environment

4. No client contacted the community hepatitis nurse within the month monitored.

Conclusions Our low cost intervention in community pharmacies to increase HCV testing Resulted in not a single PWID referring themselves. While in principle community pharmacies are willing to engage in strategies to increase HCV testing amongst PWID, this was hindered by lack of time and resources.

PWID, especially those who are actively injecting and those newly referred remain highly vulnerable and disenfranchised. This makes it unlikely that they will engage with healthcare professionals in an envirnoment that they are not comfortable with. Our data suggests that opportunist testing for PWID in pharmacies is likely to fail unless additional resources are allocated, specifically provision of education, testing, and treatment at one site and the need for dedicated individuals to deliver such a service.

\section{PTH-081 HEPATITIS E IN NORTHEAST OF ENGLAND: 5 YEAR REVIEW OF CASES AT A TERTIARY CENTRE}

Mohamed Aftab Alam*, Manoj Valappil, Stuart McPherson. Newcastle Upon Tyne Hospitals NHS Foundation Trust, Newcastle, UK

\subsection{6/gutjnl-2018-BSGAbstracts.237}

Introduction The incidence of Hepatitis E virus (HEV) infection has risen sharply in Europe in last decade, largely due to a rise in indigenous genotype 3 infections. Few studies have determined the clinical outcomes of HEV infection in the UK. Our aim was to review all cases of hepatitis $\mathrm{E}$ admitted to our unit in 5 years to determine the clinical consequences of these infections.

Methods All confirmed serological cases of HEV (IgG, IgM and RNA) between Jan 2012 and Sept 2017 were identified from our virology laboratory. Medical notes of all acute or chronic cases were reviewed retrospectively to determine epidemiological characteristics, clinical features and outcomes of the infections.

Results From a total of 206 cases had serological evidence of HEV infection, 104 were confirmed acute HEV (IgM and/or HEV RNA positive). The number of cases/year ranged from 12-27. The median age at presentation was 54 (21-94) years and $70 \%$ were $>50$ years. $68 \%$ of cases were male. $24 \%$ of acute HEV cases occurred in immunocompromised individuals. $60 \%$ of the patients developed jaundice and the median bilirubin levels was $70(4-558) \mu \mathrm{mol} / \mathrm{L}$. 2 patients had bilirubin $>500 \mu \mathrm{mol} / \mathrm{L}$. The median ALT level was 1084 (range 22-6026) U/L. Serum ALT levels $>500$ in $57 \%$ and $>5000$ in $3 \%$. No cases of fulminant liver failure were seen. 8 cases became chronic (HEV viraemia $>3$ months), all in immunocompromised individuals $(50 \%$ haematological malignancies $50 \%$ solid organ transplants). All 8 patients were treated with ribavirin with 5 (63\%) achieving sustained virological response. One patient with a delayed diagnosis of HEV developed progressive liver failure and required Liver transplantation despite ribavirin. One relapsed following 3 months ribavirin and then was a non-responder to 6 months ribavirin and 6 months PEG-interferon +ribavirin. One was non-responder to 18 months ribavirin.

Conclusion Symptomatic HEV is relatively common in the North East of England leading to jaundice and significant transaminitis in the majority. Chronic infection developed in a quarter of immunosuppressed individuals and can progress to clinically significant disease.

\section{PTH-082 PROVISION OF TIPS FOR VARICEAL HAEMORRHAGE IN NORTH EAST OF ENGLAND}

Mohamed Aftab Alam*, Fahd Rana, Jess Dyson, Ralph Jackson, Peter Littler, Rob Williams, Mark Hudson. Newcastle Upon Tyne Hospitals NHS Foundation Trust, Newcastle, UK

\subsection{6/gutjnl-2018-BSGAbstracts.238}

Introduction The North East encompasses a wide geographical area, the farthest hospital being $160 \mathrm{~km}$ from the specialist centre providing transjugular intrahepatic portosystemic shunt 